\title{
A word of caution on renal risks of prothrombin complex concentrate use in cardiac surgery
}

\author{
Christian J. Wiedermann ${ }^{*}$
}

\author{
See related research by Cappabianca et al., http://www.ccforum.com/content/20/1/5
}

In a retrospective study of bleeding patients in cardiac surgery, Cappabianca et al. [1] observed that the use of prothrombin complex concentrate (PCC) compared with fresh-frozen plasma (FFP) was associated with a higher risk of postoperative renal complications. In the unadjusted comparison, acute kidney injury (AKI) incidence was significantly higher in the PCC group, and use of PCC was an independent predictive factor of AKI development and the need for renal replacement therapy in the propensity-adjusted multivariate analysis. Since prothrombotic effects of PCC are unlikely as the underlying mechanism because an increase of thromboembolic events was not observed in the study, the authors speculate that volume excess given with FFP and a more hypovolemic balance with PCC in the context of bleeding patients could have exerted a protective effect on kidney function in the FFP group. Interestingly, in the PCC group, use of inotropes was significantly lower than in the FFP group $(p=0.007)$ [1]. This may have contributed to the observed difference in renal risk, since a recent meta-analysis on perioperative hemodynamic management associated inotropic drug use with a reduction in the incidence of postoperative AKI (odds ratio $=0.52,95 \%$ confidence interval $=0.34-0.80, p=0.003$, 14 studies, $n=1634$ patients; included were two studies in cardiac surgery, $n=433$ patients) [2]. The observed association of PCC use with higher risk of AKI may therefore be due to unknown confounders in this observational propensity score-matched analysis [3] rather than due to direct pharmacological effects of PCC.

\section{Authors' response}

\section{Giovanni Mariscalco, Daniele Maselli, Aamer B. Ahmed and Fausto Biancari}

We read with great interest this letter from Wiedermann regarding our recent study [1] investigating the safety and efficacy of PCC as first-line treatment in bleeding after cardiac surgery. In our study, we observed an increased incidence of AKI and renal replacement therapy in the group of patients receiving PCC compared with those receiving $\operatorname{FFP}(31.3 \%$ vs. $23.4 \%, p=0.01$ and $3.1 \%$ vs. $1.2 \%, p=0.037$, respectively). We originally speculated that embolic events and especially the hypovolemia accompanying PCC use could have explained the observed correlation with renal dysfunction. Wiedermann underlined that the lower AKI rate observed in the FFP group could have been related to the high rate of inotropes (protective confounders) encountered in the same patient group rather than the hypovolemic balance following PCC use. We agree that postoperative AKI is a consequence of an interplay of different pathophysiologic mechanisms, and it remains distinctly possible that inotropes may protect against AKI, ameliorating blood pressure and perfusion [4]. In addition, our study could also have reflected the inherited inability of observational investigations in proving causality. However, the correlation between inotropes and

* Correspondence: christian.wiedermann@asbz.it

Department of Internal Medicine, Central Hospital of Bolzano/Bozen, Teaching Hospital of the Medical University of Innsbruck, Lorenz-Böhler Street 5, 39100 Bolzano, Bozen (BZ), Italy 
AKI is disputed [5], and even in the meta-analysis by Prowle and colleagues [2,5] the exact AKI contribution of inotropes and volume fluid resuscitation was difficult to ascertain. Nevertheless, our study did not show any significant difference between the rate of inotropes in the PCC and FFP propensity matched groups (odds ratio $=0.74,95 \%$ confidence interval $=0.50-1.08)[1]$.

\section{Abbreviations}

AKI: acute kidney injury; FFP: fresh frozen plasma; PCC: prothrombin complex concentrate.

\section{Competing interests}

CJW has received fees for speaking and travel reimbursements from manufacturers of plasma-derived therapies (CSL Behring, Kedrion, Baxter).

\section{Authors' contributions}

CJW developed the concept for the letter and wrote the manuscript. No other individual or organization made any substantial contribution to the creation and revision of the letter. This letter is solely the work product of the author.

Published online: 17 March 2016

\section{References}

1. Cappabianca G, Mariscalco G, Biancari F, Maselli D, Papesso F, Cottini M, Crosta S, Banescu S, Ahmed AB, Beghi C. Safety and efficacy of prothrombin complex concentrate as first-line treatment in bleeding after cardiac surgery. Crit Care. 2016;20:5.

2. Prowle JR, Chua HR, Bagshaw SM, Bellomo R. Clinical review: Volume of fluid resuscitation and the incidence of acute kidney injury-a systematic review. Crit Care. 2012;16:230.

3. Austin PC, Laupacis A. A tutorial on methods to estimating clinically and policy-meaningful measures of treatment effects in prospective observational studies: a review. Int J Biostat. 2011;7:6.

4. Mariscalco G, Lorusso R, Dominici C, Renzulli A, Sala A. Acute kidney injury: a relevant complication after cardiac surgery. Ann Thorac Surg. 2011;92:1539-47.

5. Patel NN, Rogers CA, Angelini GD, Murphy GJ. Pharmacological therapies for the prevention of acute kidney injury following cardiac surgery: a systematic review. Heart Fail Rev. 2011;16:553-67. 